\title{
The clinical significance of transforming acidic coiled-coil protein 3 expression in non-small cell lung cancer
}

\author{
FENG JIANG* ${ }^{*}$, BOHUA KUANG* , YI QUE, ZHIRUI LIN, LI YUAN, WEI XIAO, \\ RUIQING PENG, XIAOSHI ZHANG and XING ZHANG \\ State Key Laboratory of Oncology in South China; Collaborative Innovation Center for Cancer Medicine, \\ Sun Yat-sen University Cancer Center, Guangzhou 510060, P.R. China
}

Received August 3, 2015; Accepted September 23, 2015

DOI: $10.3892 /$ or.2015.4373

\begin{abstract}
The relationship between TACC3, a member of the transforming acidic coiled-coil proteins (TACCs) family, and lung carcinoma remains unclear. The present study was designed to explore the prognostic and clinical significance of TACC3 in non-small cell lung cancer (NSCLC). An immunohistochemistry (IHC) assay was performed to analyze the expression of TACC3 in 195 lung cancer cases. The mRNA and protein levels of TACC3 were examined by quantitative reverse transcription-PCR or western blotting. The correlation between TACC3 expression and clinicopathological factors was analyzed by $\chi^{2}$ analysis and Fisher's exact test. Kaplan-Meier analysis and the Cox proportional hazards model were used to examine the correlation of prognostic outcomes with TACC3. The results showed that the levels of TACC3 mRNA and total protein were higher in lung cancer lesions than paired non-cancerous tissues. IHC analysis revealed that TACC3 was highly expressed in 94 (48.2\%) cases. The expression of TACC 3 was strongly correlated with smoking status, histological classification, differentiation, cytokeratin 19 fragment levels, T stage and the clinical stage of NSCLC patients. Univariate and multivariate analyses demonstrated that TACC3 is a useful biomarker for NSCLC prognosis. The low TACC3 expression group exhibited better progression-free survival (PFS) among patients who received anti-microtubule chemotherapy. In conclusion, the results showed that a high level of TACC 3 expression was correlated with advanced clinicopathological classifications, poor overall survival (OS) and poor recurrence-free survival (RFS) in NSCLC patients.
\end{abstract}

Correspondence to: Professor Xing Zhang, State Key Laboratory of Oncology in South China; Collaborative Innovation Center for Cancer Medicine, Sun Yat-sen University Cancer Center, Guangzhou 510060, P.R. China

E-mail: zhangxing@sysucc.org.cn; zhangxng@mail.sysu.edu.cn

*Contributed equally

Key words: non-small cell lung cancer, immunohistochemistry, TACC3, prognosis, chemotherapy sensitivity
Our findings indicate that TACC3 is a potential prognostic marker and therapeutic target for NSCLC.

\section{Introduction}

Lung cancer is the most common cause of cancer mortality globally and the second most common type of cancer (1). The high mortality associated with lung cancer is primarily attributable to late diagnosis and metastasis at the time of diagnosis. Identifying effective tumor biomarkers of this malignancy in the early stages and preventing metastasis may therefore be the best options for improving the generally dismal survival rate of lung cancer patients.

Structural and numerical abnormalities of the centrosome are significantly associated with chromosome instability, aneuploidy and tumorigenesis $(2,3,4-8)$. The transforming acidic coiled-coil proteins (TACCs) family is characterized by a highly conserved carboxyl-terminus coiled-coil 'TACC domain', which is important for the interaction with spindle/centrosome dynamics $(2,4,9)$. The TACC family contains TACC1, TACC 2 and TACC 3 in mammals $(2-4,10)$. Although increasing evidence indicates that abnormalities in human TACCs (TACC1-3) may correlate with the development of some human malignancies, the functions of these three proteins in mammals are largely unknown (11-13).

The human TACC1-3 genes are located on chromosome $8 \mathrm{p} 11,10 \mathrm{q} 26$ and $4 \mathrm{p} 16.3$, respectively $(2,4,11-17)$. TACC3 is mainly expressed in the developing embryo and in adult testis, thymus, peripheral blood leukocytes, spleen and intestinal tissues $(4,17)$. Several studies had shown that TACC3 may be associated with some human tumor, but the expression status of TACC 3 in cancer remains controversial and unclear. For instance, a microarray analysis suggested that TACC3 is upregulated during the transition of ductal carcinoma in situ to invasive ductal carcinoma, while an immunohistochemical analysis demonstrated that TACC3 is downregulated in resected breast tumors $(18,19)$. A similar discrepancy was found in the expression of TACC 3 in ovarian tumor and thyroid cancer (20-22). In addition, TACC 3 has been identified as a potential glioblastoma multiforme oncogene and may be overexpressed in multiple myeloma cases and lung cancer (23-25). Therefore, it is necessary to explore the expression status of TACC 3 in lung cancer and the relationship between TACC 3 
expression and clinicopathological characteristic of lung cancer.

In the present study, we focused on the expression patterns of TACC3 in primary non-small cell lung cancer (NSCLC) lesions and metastatic lymph nodes. Statistical analysis indicated a relationship between high TACC3 expression levels and the clinicopathological features of NSCLC. Multivariate Cox regression analyses suggested that higher TACC3 expression in NSCLC predicts poorer survival independent of other factors.

\section{Materials and methods}

Patients and tumor specimen. The present study is reported according to the Reporting Recommendations for Tumor Marker Prognostic Studies (REMARK) (26). A total of 207 consecutive cases of primary NSCLC with surgical resection were collected from Sun Yat-sen University Cancer Center (SYSUCC; Guangzhou, China) between January 2003 and June 2004. Fig. 1 shows the inclusion and exclusion criteria for the present study. Ultimately, a total of 195 patients were enrolled.

We evaluated conventional clinical characteristics as shown in Table I. All 195 cases were reevaluated with respect to histological subtype, differentiation and tumor stage. The follow-up interval ranged from 0.9 to 139.4 months (median 27.3 months, mean 49.5 months). The 5-year cumulative survival rate for all patients was $30 \%$.

A total of 195 paraffin-embedded NSCLC tissue samples and 11 matched adjacent paraffin-embedded non-cancerous human lung tissues were obtained from the archives of the Department of Sample Resources, SYSUCC. These samples had been histologically and clinically diagnosed. Fifty metastatic lymph nodes were also obtained from the above-mentioned patients. Eleven paired freshly frozen lung carcinoma and non-cancerous tissues adjacent to cancer lesions were obtained from the same patients. The present study was conducted with the consent of the patients and the approval of the Institute Research Ethics Committee of SYSUCC.

Immunohistochemistry. Immunohistochemistry was performed as previously described (27). The relevant, sections were incubated with an anti-TACC3 monoclonal antibody (1:800; Abcam, Cambridge, UK) at $4^{\circ} \mathrm{C}$ overnight. Immunohistochemical kit (SP-9001 rabbit SP kit, lot: 50581654) was obtained from Zhongshan Golden Bridge Biotechnology Co. Ltd. (Beijing, China). As a negative control, the primary antibody was replaced by $\mathrm{IgG}$.

The degree of immunostaining was examined and evaluated independently by two pathologists without knowledge of the clinical characteristics of the cases. The proportion of positively stained tumor cells varied from 0 to $100 \%$, and the intensity of staining varied from weak to strong. The proportion of stained tumor cells was graded according to the following criteria: 0 (no positive tumor cells), 1 ( $\leq 10 \%$ positive tumor cells), 2 (11-25\% positive tumor cells), 3 (26-40\% positive tumor cells) and 4 ( $\geq 41 \%$ positive tumor cells). The intensity of staining was quantified using the following score system: 0 (no staining), 1 (weak staining, light yellow), 2 (moderate staining, yellowish brown) and 3 (intense staining, brown). The TACC3

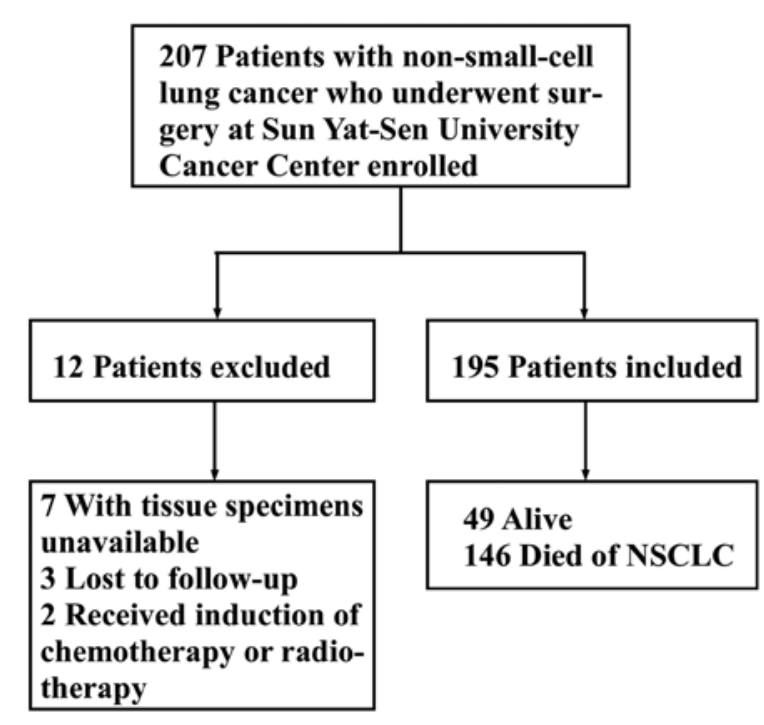

Figure 1. Inclusion and exclusion criteria for the present study.

expression index was calculated by multiplying the two scores to obtain a final score of $0,1,2,3,4,6,9$ or 12 . The cut-off value for high and low levels of expression was based on a measurement of heterogeneity using a log-rank test statistical analysis with respect to the overall survival (OS) rate. The carcinomas were finally classified as low expression (score $<5$ ) or high expression (score $\geq 6$ ).

Western blotting. Western blot analysis was performed as previously described (27). The membrane was incubated with primary antibodies $(1: 1,000)$ at $4^{\circ} \mathrm{C}$ overnight, followed by incubation with horseradish peroxidase-conjugated goat antimouse or anti-rabbit IgG secondary antibody $(1: 3,000)$ (both from Abcam) at room temperature for $1 \mathrm{~h}$. A mouse $\beta$-actin monoclonal antibody (1:4,000; Cell Signaling Technology, Danvers, MA, USA) was used as an internal loading control. The membrane was detected using an enhanced chemiluminescence (ECL; Amersham Pharmacia Biotech, UK) detection system (Amersham Biosciences Europe, Freiburg, Germany) according to the manufacturer's instructions.

RNA extraction and quantitative reverse transcription-polymerase chain reaction ( $R T-P C R)$ analysis. Total RNA was extracted from 11 paired freshly frozen lung cancer and noncancerous tissues using TRIzol reagent (Invitrogen Life Technologies, Carlsbad, CA, USA) according to the manufacturer's instructions. The RNA was treated with RNA-free DNase, and $2.0 \mu \mathrm{g}$ of total RNA was used to generate cDNA using the RETROscript kit (Promega, Madison, WI, USA). The full-length open reading frame of TACC3 was PCR amplified from cDNA samples. RT-PCR was performed using iQ SYBR-Green Supermix on a MYiQ qPCR machine (both from Bio-Rad, Hercules, CA, USA). The following primers were used to amplify TACC3: forward, 5'-CCTCTTCAAG CGTTTTGAGAAAC-3' and reverse, 5'-GCCCTCCTGGGT GATCCTT-3'. The following primers were used to amplify $\beta$-actin: forward, 5'-GACTCATGACCACAGTCCATGC-3' and reverse, 5'-AGAGGCAGGGATGATGTTCTG-3'. The 
Table I. Correlation between TACC3 expression and clinicopathological characteristics.

TACC3 expression: no. of patients (\%)

Characteristics

$\operatorname{Low}^{\mathrm{a}}(101)$

$\operatorname{High}^{\mathrm{a}}(94)$

P-value

Gender

Male

76 (49.7)

77 (50.3)

0.258

Female

$25(59.5)$

$17(40.5)$

Age (years)

$\leq 60$

$54(50.5)$

$53(49.5)$

0.682

$>60$

47 (53.4)

41 (46.6)

Smoking status ${ }^{\mathrm{c}}$

No

$40(63.5)$

23 (36.5)

0.027

Yes

61 (46.6)

70 (53.4)

Differentiation

Well-moderate

53 (63.9)

30 (36.1)

Poor

48 (42.9)

64 (57.1)

T stage $^{\mathrm{d}}$

T1

23 (69.7)

10 (30.3)

0.024

T2-4

78 (48.1)

84 (51.9)

$\mathrm{N}$ stage ${ }^{\mathrm{d}}$

N0

54 (56.8)

41 (43.2)

0.168

$\mathrm{N} 1-3$

45 (46.9)

$51(53.1)$

M stage ${ }^{\mathrm{d}}$

M0 $82(49.7)$

$83(50.3)$

0.169

M1

$19(63.3)$

$11(36.7)$

Clinical stage $\mathrm{d}^{\mathrm{d}}$

I

$36(66.7)$

18 (33.3)

0.001

II

$18(32.1)$

38 (67.9)

III

$28(50.0)$

$28(50.0)$

$19(65.5)$

$10(34.5)$

Histological type

Adenocarcinoma

67 (59.3)

46 (40.7)

34 (41.5)

48 (58.5)

$\mathrm{SCCA}^{\mathrm{e}}$

49 (53.8)

42 (46.2)

$6(40.0)$

$9(60.0)$

(+)

40 (55.6)

32 (44.4)

27 (47.4)

(+)

30 (52.6)

0.004

0.004

0.320

0.741

CYFRA 21-1 ${ }^{\mathrm{g}}$
$4(26.7)$
13 (65.0)
$11(73.3)$
$7(35.0)$
(t)

TACC3, transforming acidic coiled-coil protein 3; NSCLC, non-small-cell lung cancer; SCCA, squamous cell carcinoma antigen; CEA, carcinoembryonic antigen; CYRFA 21-1, cytokeratin 19 fragment; ${ }^{a}$ TACC3 low expression, score <5; high expression, score $\geq 6$; ${ }^{b}$ two-sided $\mathrm{P}$-values were calculated using Pearson's $\chi^{2}$ test or continuity correction to evaluate the significance of the correlations. Bold print indicates statistical significance. ${ }^{~}$ Smoking status is unknown for 1 patient. ${ }^{\mathrm{d}}$ Tumor size, lymph node involvement, distant metastasis and clinical stage were classified or reclassified according to the seventh edition of the International Union Against Cancer (UICC) staging system for Lung Cancer. N stage is unknown for 4 patients. ${ }^{\text {SCCA: }}-$, normal $(0-1.50 \mathrm{ng} / \mathrm{ml})$; +, elevated $(>1.50 \mathrm{ng} / \mathrm{ml})$; SCCA unknown for 89 patients. ${ }^{\mathrm{f}}$ CEA: -, normal $(0-5 \mathrm{ng} / \mathrm{ml})$; +, elevated (>5 ng/ml); CEA unknown for 66 patients. gCYFRA 21-1: -, normal $(0.1-3.30 \mathrm{ng} / \mathrm{ml})$; +, elevated (>3.30 ng/ml); CYFRA 21-1 unknown for 160 patients.

primer sets were designed and produced by PrimerDesign Ltd. (Southampton, UK). Cycles were as follows: $95^{\circ} \mathrm{C}$ for $3 \mathrm{~min}$
(1 cycle), $94^{\circ} \mathrm{C}$ for $30 \mathrm{sec}$ followed by $60^{\circ} \mathrm{C}$ for $10 \mathrm{sec}$ and $72^{\circ} \mathrm{C}$ for $15 \mathrm{sec}\left(40\right.$ cycles), and $95^{\circ} \mathrm{C}$ for $30 \mathrm{sec}(1$ cycle). Melt curve 
A
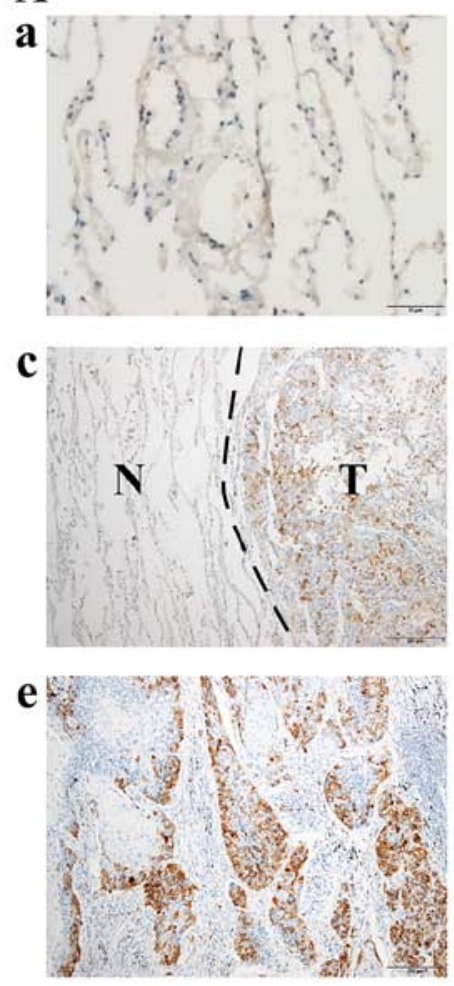
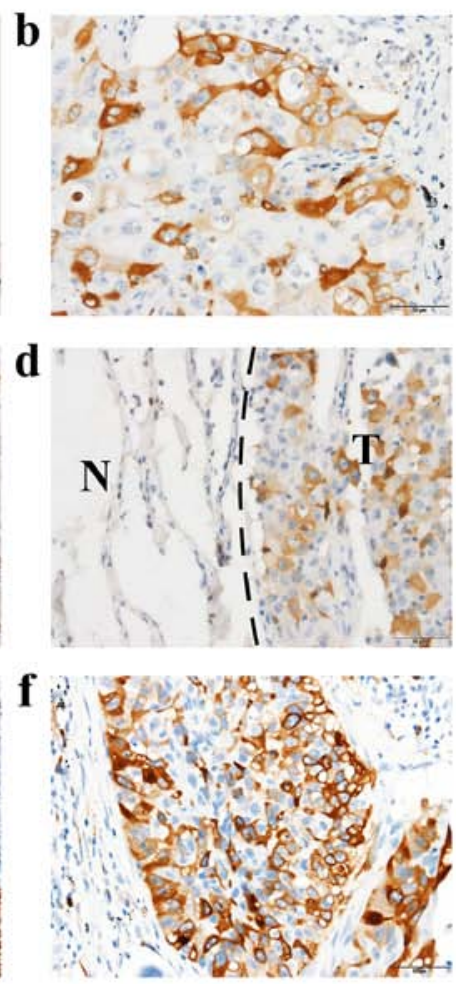

B

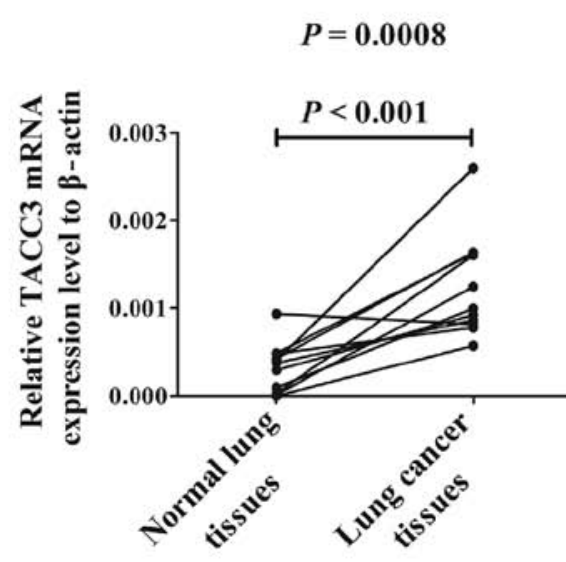

Paired lung cancer tissues

C

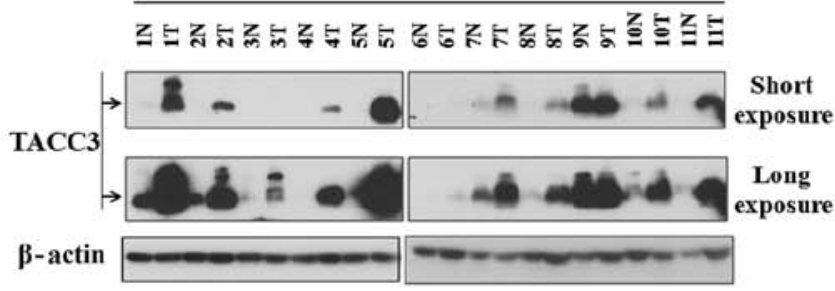

Figure 2. The expression of TACC3 is elevated in fresh frozen primary NSCLC tissues compared with non-cancerous tissues adjacent to the cancer lesions. (A) Expression of TACC3 in different tissues as determined by immunohistochemical staining. a, Lack of TACC3 staining in the alveolar epithelium of a normal lung (magnification, $\mathrm{x} 400$ ). b, Cytoplasmic TACC3 staining in primary lung cancer lesions (magnification, x400). c and d, Higher expression of the TACC3 protein in primary lung cancer lesions compared with adjacent non-cancerous tissues (c, magnification, $\mathrm{x} 100$; $\mathrm{d}$, magnification, $\mathrm{x} 400)$. e and $\mathrm{f}, \mathrm{Strong}$ cytoplasmic TACC3 staining in the matched metastatic lymph nodes of adenocarcinomas (e, magnification, x100; f, magnification, $\mathrm{x} 400$ ). (B) Expression analysis of TACC 3 mRNA in paired and fresh frozen primary NSCLC (T) and non-cancerous tissues (N) from the same patient by real-time RT-PCR. $\beta$-actin was used as an internal control. The reactions were performed in triplicate in three independent experiments. (C) Expression analysis of TACC 3 protein in paired and fresh frozen primary NSCLC tissues (T) and non-cancerous tissues (N) from the same patient by western blotting. $\beta$-actin was used as an internal reference.

analysis was performed for each reaction to ensure amplification specificity.

Statistical analysis. All statistical analyses were performed using the SPSS version 16.0 statistical software package (SPSS, Inc., Chicago, IL, USA).

The data are presented as the mean \pm SEM unless otherwise indicated. Differences among variables were identified by $\chi^{2}$ analysis or two-tailed Student's t-tests. Correlations between TACC 3 expression and clinicopathological factors were assessed by $\chi^{2}$ analysis and Fisher's exact test. Survival curves were plotted using Kaplan-Meier survival analysis and compared using a log-rank test. Further analyses of survival curves were calculated based on stratifying age, TNM classifications, histological type and clinical stages. Univariate and multivariate Cox proportional hazards models were used to evaluate the relative risks (RRs) of death associated with TACC3 expression and other variables. In our analyses, we defined the RR of 1.000 as baseline for factors including gender (male), age ( $\leq 60$ years), smoking status (non-smoker), differentiation (well-moderate), SCC (0-15.0 ng/ml), T1, N0, M0, clinical stage (I-II), and low level of TACC3 expression. In all cases, $\mathrm{P}<0.05$ was considered to indicate a statistically significant result.

\section{Results}

Immunohistochemistry reveals high TACC3 expression in NSCLC tissue samples. As shown inFig. 2A, normal lung alveolar epithelium did not express TACC3 protein (Fig. 2A-a, -c and -d). TACC3 immunoreactivity was generally confined to the cytoplasm of tumor cells in NSCLC lesions and metastatic lymph nodes (Fig. 2A-b, -c, -d, -e and -f). In all NSCLC tissue samples, TACC3 protein was observed in 180 of 195 (92.3\%) human NSCLC samples, and strong cytoplasmic staining of TACC3 protein was detected in $64(35.5 \%)$ tumors. Among the corresponding metastatic lymph nodes, 47 of 50 (94\%) cases displayed positive staining (Fig. 2A-e and -f), and 48.9\% (23 of 47) of cases exhibited strong staining.

TACC3 expression is upregulated in NSCLC tissue samples as determined by real-time RT-PCR and western blotting. To investigate the mRNA and protein expression levels of TACC3 in NSCLC lesions, real-time RT-PCR and western blot analyses were performed in paired freshly frozen NSCLC tissues and adjacent non-cancerous tissues from the same patients. As determined by real-time RT-PCR analysis, all 11 human NSCLC samples exhibited higher TACC3 mRNA expression compared to paired non-cancerous tissues (Fig. 2B). Western 

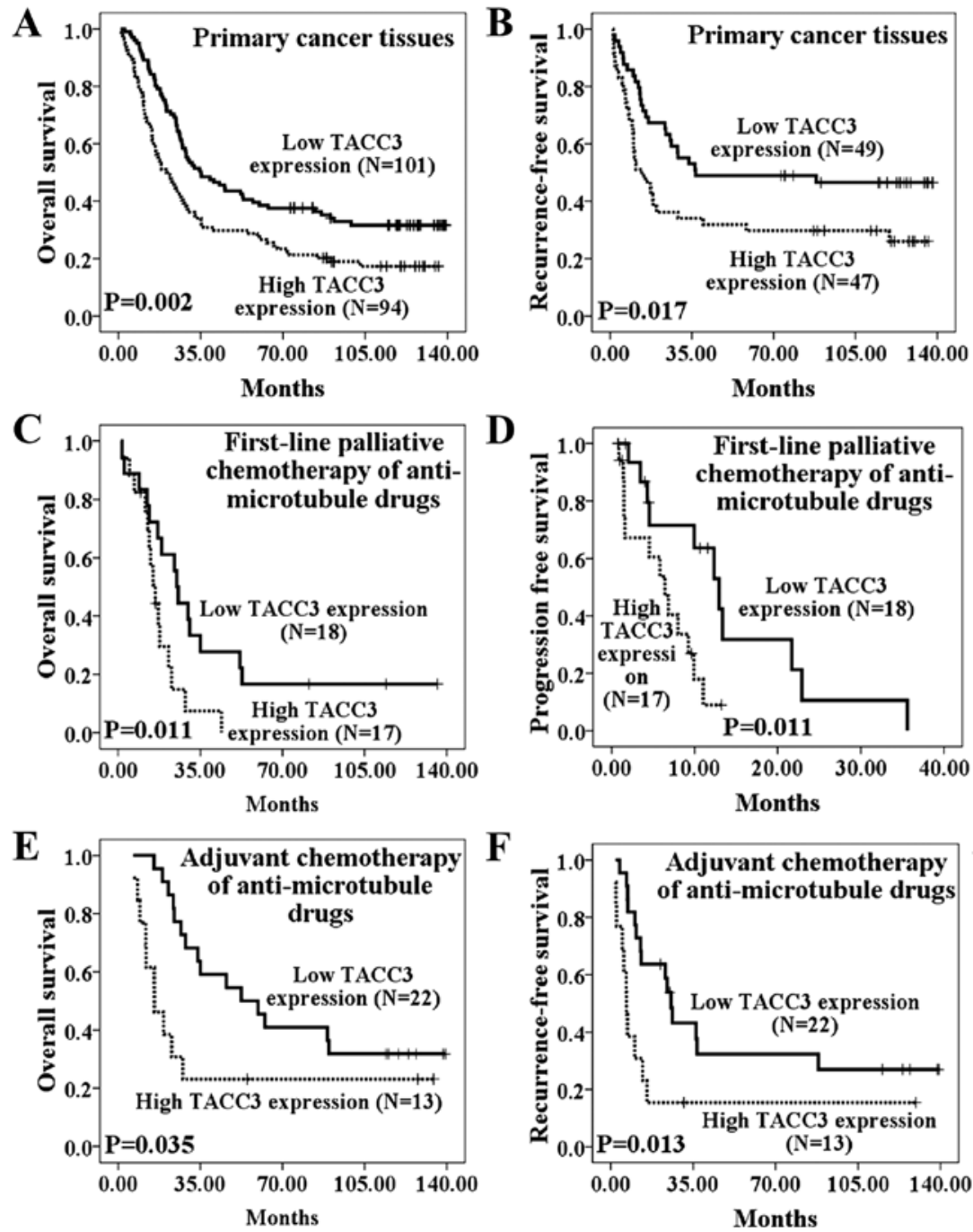

Figure 3. Influence of TACC 3 expression on NSCLC patient survival and TACC3 expression levels are correlated with sensitivity to anti-microtubule drugs (A) OS curves and analysis of 195 primary NSCLC tissues demonstrated that patients with high TACC3 expression have short survival times ( $\mathrm{P}=0.002$ ). (B) The duration of RFS differed significantly between patients with high and low TACC 3 expression $(\mathrm{P}=0.017)$, with better survival in the low TACC 3 expression group. TACC 3 low expression, score $<5$; high expression, score $\geq 6$. (C and D) The low TACC 3 expression group exhibited better overall survival $(\mathrm{P}=0.011)$ and progression-free survival $(\mathrm{P}=0.011)$ among patients who received anti-microtubule chemotherapy as a first-line palliative therapy. (E and $\mathrm{F})$ Among patients who received anti-microtubule chemotherapies as adjuvant chemotherapy, low TACC 3 expression was also correlated with longer overall survival time $(\mathrm{P}=0.035)$ and longer recurrence-free survival time $(\mathrm{P}=0.013)$ compared with patients expressing high TACC3 levels.

blot analysis demonstrated that TACC3 protein was markedly overexpressed in the NSCLC lesions but weakly detected in adjacent paired non-cancerous tissues (Fig. 2C).

Correlation between the clinicopathological features and TACC 3 expression levels. The immunoreactivity results of TACC3 expression levels were statistically analyzed to determine their relationships with the clinical characteristics of the NSCLC patients. As presented in Table I, the expression of TACC3 was strongly correlated with smoking status, histological classification, differentiation, CYFRA21-1 levels, $\mathrm{T}$ stage and the clinical stage of NSCLC patients. However, there was no significant correlation between the expression level of TACC 3 protein and gender, age, squamous cell carcinoma antigen (SCCA), carcinoembryonic antigen (CEA), tumor supplies group of factors (TSGF), N classification or distant metastasis of NSCLC patients.
TACC3 expression is correlated with poor overall and recurrence-free survival. After demonstrating the correlation of TACC3 expression with clinicopathological characteristics, we proceeded to examine the relationship between TACC3 expression level and the patients survival. The patients OS time was defined as the time from the day of surgery to (i) the point of death due to recurrence or metastasis or (ii) to the end point of the most recent follow-up if the patient was alive. Recurrence-free survival (RFS) was calculated from the date of curative surgery to the day of disease recurrence, disease progression, death or the most recent follow-up. Patients with metastases were excluded from the RFS analysis.

The relationship between TACC 3 expression levels and OS time $(\mathrm{P}=0.002)$ is presented in Fig. 3A. In primary NSCLC lesions, the low TACC3 expression group had better OS (median, 35.10 months), whereas the high TACC3 expression group had poor OS (median, 20.33 months). 

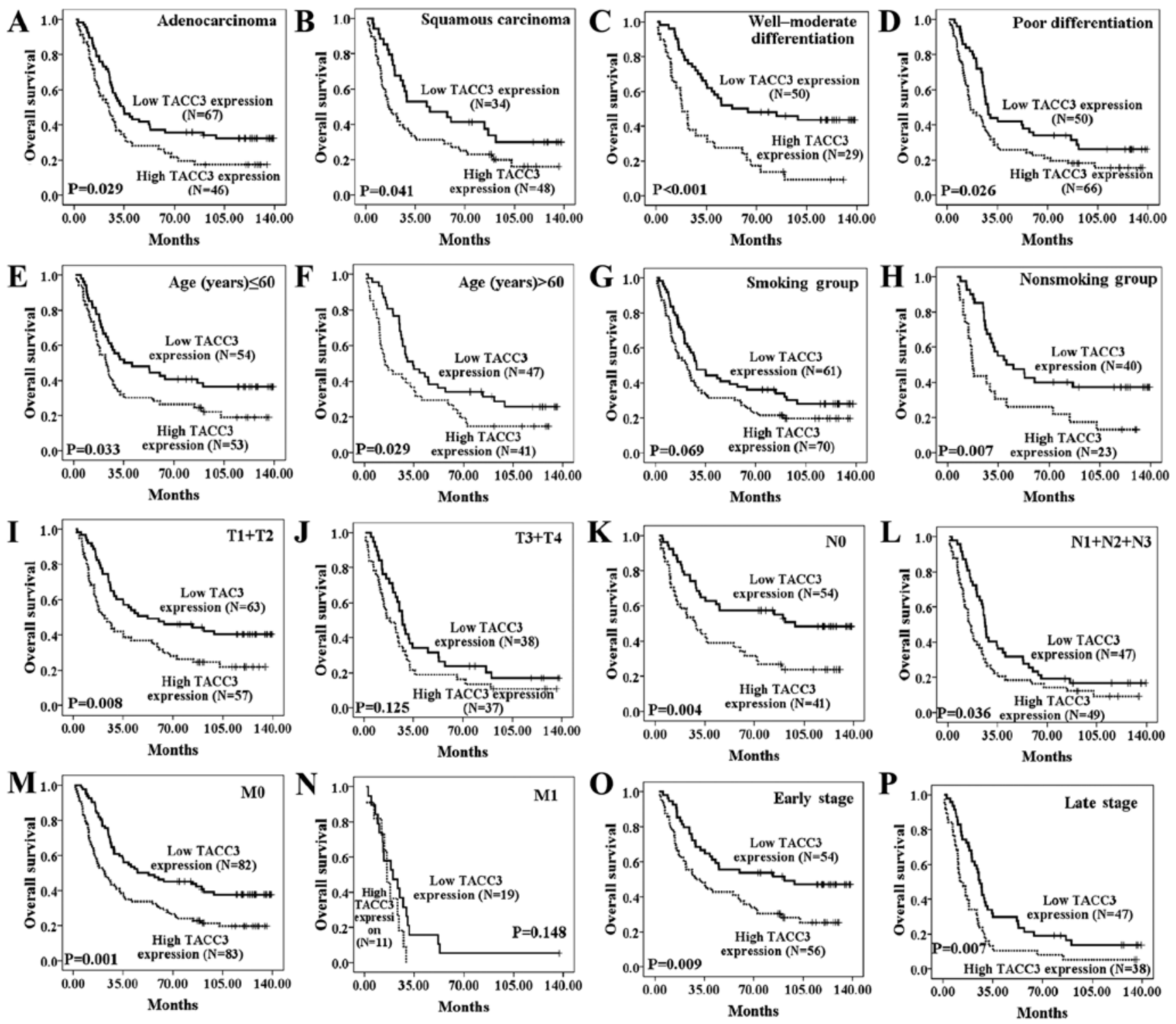

Figure 4. The influence of TACC 3 expression on overall survival of NSCLC patients stratified by clinicopathological characteristics. Kaplan-Meier curves were stratified by TACC 3 expression level according to histological type, tumor differentiation, age, smoking status, as well as T, N, M and clinical stage (A-P). Patients with high TACC3 expression had poor OS regardless of histological type (A and B), tumor differentiation (C and D), age (E and F), N stage (K and L) and clinical stage $(\mathrm{O}$ and $\mathrm{P})$. Patients with high TACC3 expression have poorer OS in the non-smoking group (H), early T stage (T1+T2) (K), and M0 stage (M) subgroups. TACC 3 low expression, score $<5$; high expression, score $\geq 6$. Smoking status is unknown for 1 patient. $\mathrm{N}$ stage is unknown for 4 patients.

The cumulative 5-year survival rate was $38.6 \%$ in the low TACC 3 expression group and $25.5 \%$ in the high TACC 3 expression group. Furthermore, patients with a low TACC3 expression had better OS regardless of the patients age, histological type, tumor differentiation, $\mathrm{N}$ stage and clinical stage. Moreover, the OS showed a statistically significant difference between the high and low TACC 3 expression groups in the non-smoking group, early $\mathrm{T}$ stage $(\mathrm{T} 1+\mathrm{T} 2)$ and M0 stage subgroups (Fig. 4A-P).

As shown in Fig. 3B, RFS also differed significantly between patients with high and low TACC 3 expression levels $(\mathrm{P}=0.017)$. RFS also significantly differed between the high and low TACC 3 expression groups in the younger age group ( $\leq 60$ years), among cases with well-moderated differentiation, and in the early T stage (T1+T2), N0 and M0 stages, and early clinical stage (I+II) subgroups (Fig. 5A-O).
Univariate and multivariate analyses indicate an independent role of TACC3 in the prognosis of NSCLC. As shown in Tables II and III, univariate Cox regression analyses revealed that a higher level of TACC 3 was associated with a significantly increased risk of cancer-related death $(\mathrm{P}=0.002$, $\mathrm{RR}=1.663 ; \mathrm{P}=0.019, \mathrm{RR}=1.849$ ) in NSCLC patients. The RRs indicated that the level of SCCA, differentiation and clinical staging were also effective predictors. Multivariate Cox regression analyses suggested that higher TACC 3 expression in NSCLC predicted worse survival independent of other factors $(\mathrm{P}=0.005, \mathrm{RR}=1.999 ; \mathrm{P}=0.022, \mathrm{RR}=1.825)$. As expected, clinical staging was also recognized as independent prognostic factors.

Taken together, the univariate and multivariate analyses suggested that TACC3 may be a useful biomarker for the prognosis of NSCLC patients. 

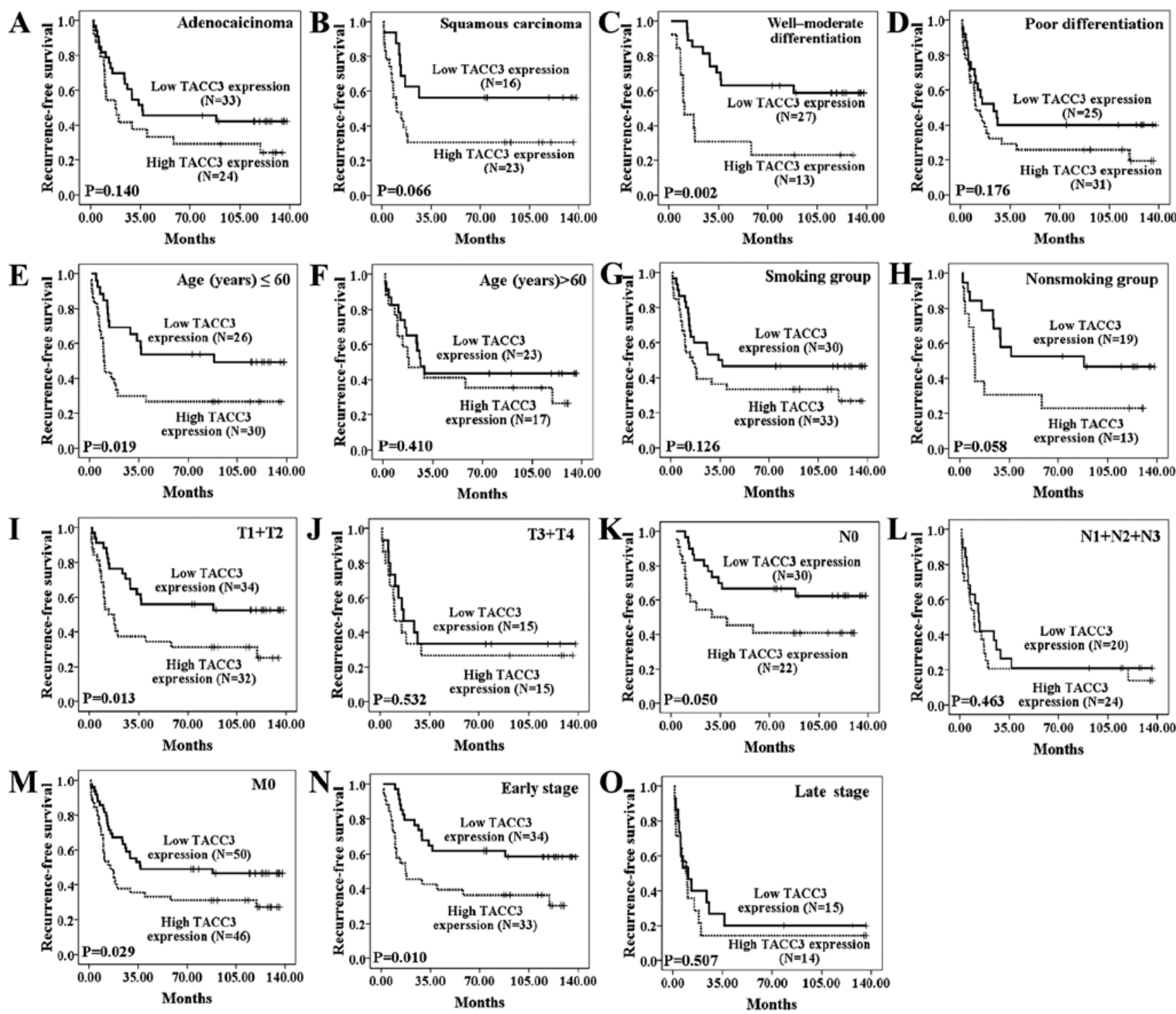

Figure 5. The influence of TACC3 expression on recurrence-free survival of NSCLC patients stratified by clinicopathological characteristics. (A and B) The duration of RFS did not differ significantly between the two groups among the histological type subgroups $(\mathrm{P}=0.140$ and $\mathrm{P}=0.066)$. In addition, the duration of RFS differed significantly between the high and low TACC 3 expression groups among the well-moderate differentiation $(\mathrm{C})$, younger age $(\leq 60$ years) (E), early T stage (T1+T2) (I), N0 stage (K), M0 stage (M), and early clinical stage (I+II) (N) subgroups. The duration of RFS did not differ significantly between the high and low TACC3 expression groups among the poor differentiation (D), older age ( $>60$ years) (F), smoking $(\mathrm{G})$ or non-smoking groups (H), or the late $\mathrm{T}$ stage $(\mathrm{T} 3+\mathrm{T} 4)(\mathrm{J})$, advanced $\mathrm{N}$ stage $(\mathrm{N} 1+\mathrm{N} 2+\mathrm{N} 3)$ (L) and late clinical stage (III+IV) (O) subgroups. TACC3 low expression, score $<5$; high expression, score $\geq 6$.

TACC3 expression levels are correlated with sensitivity to anti-microtubule drugs. As shown in Fig. 6, 35 patients received adjuvant chemotherapy with platinum drugs (cisplatin or carboplatin) and anti-microtubule drugs, including paclitaxel (16 patients), docetaxel (7 patients) and vinorelbine (12 patients). Among the patients who progressed after adjuvant chemotherapy and those who received palliative therapy, 35 received first-line palliative therapy with platinum and anti-microtubule drugs, including paclitaxel (20 patients), docetaxel (6 patients) and vinorelbine (9 patients).

OS of patients who received chemotherapy was defined as the time from the day of the first chemotherapy treatment to (i) the point of death due to recurrence or metastasis or (ii) to the end point of the most recent follow-up if the patient was alive. Progression-free survival (PFS) of patients who received palliative chemotherapy was defined as the interval between the first chemotherapy treatment and disease progression or last contact. RFS of patients who received adjuvant chemotherapy was calculated from the date of the first chemotherapy treatment to the day of disease recurrence, disease progression, death or the most recent follow-up.

To explore whether expression of TACC 3 was correlated with sensitivity to anti-microtubule drugs, we used KaplanMeier analysis and log-rank tests to examine the relationship between the TACC 3 expression level and survival in patients who received anti-microtubule chemotherapy. As shown in Fig. 3C and D, the low TACC3 expression group exhibited better OS $(\mathrm{P}=0.011)$ and better PFS $(\mathrm{P}=0.011)$ among patients who received anti-microtubule chemotherapy as a first-line palliative therapy. Among patients who received anti-micro- 
Table II. Univariate and multivariate Cox regression analysis of potential prognostic parameters for NSCLC patients regarding overall survival.

\begin{tabular}{|c|c|c|c|c|}
\hline & \multicolumn{2}{|c|}{ Univariate analysis } & \multicolumn{2}{|c|}{ Multivariate analysis } \\
\hline & RR $(95 \% \mathrm{CI})$ & P-value & RR (95\% CI) & P-value \\
\hline \multicolumn{5}{|l|}{ Gender } \\
\hline Male & 1.000 & 0.108 & & \\
\hline Female & $0.716(0.477-1.077)$ & & & \\
\hline \multicolumn{5}{|l|}{ Age, years } \\
\hline$\leq 60$ & 1.000 & 0.441 & & \\
\hline$>60$ & $1.137(0.820-1.575)$ & & & \\
\hline \multicolumn{5}{|l|}{ Smoking status } \\
\hline No & 1.000 & 0.186 & & \\
\hline Yes & $1.268(0.891-1.804)$ & & & \\
\hline \multicolumn{5}{|l|}{ Histology } \\
\hline Adenocarcinoma & 1.000 & 0.475 & & \\
\hline Squamous carcinoma & $1.132(0.806-1.589)$ & & & \\
\hline \multicolumn{5}{|l|}{ Differentiation } \\
\hline Well-moderate & 1.000 & 0.077 & & \\
\hline Poor & $1.376(0.966-1.961)$ & & & \\
\hline \multicolumn{5}{|l|}{$\mathrm{SCCA}^{\mathrm{a}}$} \\
\hline$(-)$ & 1.000 & 0.006 & 1.000 & 0.009 \\
\hline$(+)$ & $2.336(1.280-4.264)$ & & $2.304(1.233-4.304)$ & \\
\hline \multicolumn{5}{|l|}{$\mathrm{CEA}^{\mathrm{b}}$} \\
\hline$(-)$ & 1.000 & 0.082 & & \\
\hline$(+)$ & $1.433(0.955-2.150)$ & & & \\
\hline \multicolumn{5}{|l|}{ CYFRA $21-1^{\mathrm{c}}$} \\
\hline$(-)$ & 1.000 & 0.753 & & \\
\hline$(+)$ & $1.142(0.499-2.611)$ & & & \\
\hline \multicolumn{5}{|l|}{ Clinical stage $^{\mathrm{d}}$} \\
\hline I-II & 1.000 & $<0.001$ & 1.000 & $<0.001$ \\
\hline III-IV & $2.269(1.629-3.161)$ & & $3.255(1.990-5.323)$ & \\
\hline \multicolumn{5}{|l|}{ TACC3 expression ${ }^{\mathrm{e}}$} \\
\hline Low & 1.000 & 0.002 & 1.000 & 0.005 \\
\hline High & $1.663(1.199-2.306)$ & & $1.999(1.231-3.248)$ & \\
\hline
\end{tabular}

TACC3, transforming acidic coiled-coil protein 3; NSCLC, non-small cell lung cancer; SCCA, squamous cell carcinoma antigen; CEA, carcinoembryonic antigen; CYRFA 21-1, cytokeratin 19 fragment; TSGF, tumor-specific growth factor; CI, confidence interval; ${ }^{a}$ SCCA: -, normal $(0-1.50 \mathrm{ng} / \mathrm{ml}) ;+$, elevated $(>1.50 \mathrm{ng} / \mathrm{ml})$; SCC unknown for 89 patients. ${ }^{\mathrm{b}} \mathrm{CEA}:-$, normal $(0-5 \mathrm{ng} / \mathrm{ml}) ;+$, elevated $(>5 \mathrm{ng} / \mathrm{ml})$; CEA unknown for 66 patients. ${ }^{\circ}$ CYFRA $21-1$ : -, normal $(0.1-3.30 \mathrm{ng} / \mathrm{ml}) ;+$, elevated (>3.30 ng/ml); CYFRA 21-1 unknown for 110 patients. ${ }^{\mathrm{d}}$ Tumor staging was classified or reclassified according to the seventh edition of the International Union Against Cancer (UICC) staging system for

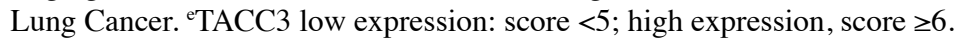

tubule chemotherapies as adjuvant chemotherapy, low TACC3 expression was also correlated with longer OS time (Fig. 3E, $\mathrm{P}=0.035$ ) and longer RFS time (Fig. $3 \mathrm{~F}, \mathrm{P}=0.013$ ) compared with patients expressing high TACC3 levels.

\section{Discussion}

Members of the TACC family play important roles in cell growth, differentiation and gene regulation by affecting centrosome/spindle dynamics $(10,17,28-29)$. In addition, the deregulation of human TACCs (TACC1-3) may be involved in the development and progression of some human malignancies $(3,10,13,15,25,30,31)$.

A previous immunohistochemical analysis of lung cancer showed TACC3 expression is higher in primary lung cancer tissues than in normal lung tissues and high level of TACC3 is associated with a poor prognosis (25). However, that study did not use western blot analysis and real-time RT-PCR to verify the conclusion and did not analysis the expression of TACC3 in metastatic lymph node tissues. In the present study, we performed western blot analysis and real-time RT-PCR as well as immunohistochemical analysis to determine whether 
Table III. Univariate and multivariate Cox regression analysis of potential prognostic parameters for NSCLC patients regarding recurrence-free survival.

\begin{tabular}{|c|c|c|c|c|}
\hline & \multicolumn{2}{|c|}{ Univariate analysis } & \multicolumn{2}{|c|}{ Multivariate analysis } \\
\hline & RR $(95 \%$ CI $)$ & P-value & RR (95\% CI) & P-value \\
\hline \multicolumn{5}{|l|}{ Gender } \\
\hline Male & 1.000 & 0.361 & & \\
\hline Female & $0.751(0.406-1.389)$ & & & \\
\hline \multicolumn{5}{|l|}{ Age, years } \\
\hline$\leq 60$ & 1.000 & 0.802 & & \\
\hline$>60$ & $0.973(0.560-1.565)$ & & & \\
\hline \multicolumn{5}{|l|}{ Smoking status } \\
\hline No & 1.000 & 0.849 & & \\
\hline Yes & $1.054(0.651-1.807)$ & & & \\
\hline \multicolumn{5}{|l|}{ Histology } \\
\hline Adenocarcinoma & 1.000 & 0.575 & & \\
\hline Squamous carcinoma & $1.127(0.669-1.896)$ & & & \\
\hline \multicolumn{5}{|l|}{ Differentiation } \\
\hline Well-moderate & 1.000 & 0.016 & 1.000 & 0.297 \\
\hline Poor & $1.911(1.128-3.235)$ & & $1.336(0.760-2.456)$ & \\
\hline \multicolumn{5}{|l|}{$\mathrm{SCCA}^{\mathrm{a}}$} \\
\hline$(-)$ & 1.000 & 0.122 & & \\
\hline$(+)$ & $2.136(0.737-6.270)$ & & & \\
\hline \multicolumn{5}{|l|}{$\mathrm{CEA}^{\mathrm{b}}$} \\
\hline$(-)$ & 1.000 & 0.302 & & \\
\hline$(+)$ & $1.329(0.743-2.611)$ & & & \\
\hline \multicolumn{5}{|l|}{ CYFRA $21-1^{c}$} \\
\hline$(-)$ & 1.000 & 0.706 & & \\
\hline$(+)$ & $1.709(0.167-3.336)$ & & & \\
\hline \multicolumn{5}{|l|}{ Clinical stage $^{\mathrm{d}}$} \\
\hline I-II & 1.000 & $<0.001$ & 1.000 & $<0.001$ \\
\hline III-IV & $2.640(1.566-4.451)$ & & $2.614(1.560-4.408)$ & \\
\hline \multicolumn{5}{|l|}{ TACC 3 expression ${ }^{\mathrm{e}}$} \\
\hline Low & 1.000 & 0.019 & 1.000 & 0.022 \\
\hline High & $1.849(1.107-3.086)$ & & $1.825(1.093-3.047)$ & \\
\hline
\end{tabular}

TACC3, transforming acidic coiled-coil protein 3; NSCLC, non-small cell lung cancer; SCCA, squamous cell carcinoma antigen; CEA, carcinoembryonic antigen; CYRFA 21-1, cytokeratin 19 fragment; TSGF, tumor-specific growth factor; CI, confidence interval; ${ }^{a}$ SCCA: -, normal $(0-1.50 \mathrm{ng} / \mathrm{ml})$; +, elevated $(>1.50 \mathrm{ng} / \mathrm{ml})$; SCC unknown for 89 patients. ${ }^{\mathrm{b}} \mathrm{CEA}:-$, normal $(0-5 \mathrm{ng} / \mathrm{ml}) ;+$, elevated $(>5 \mathrm{ng} / \mathrm{ml})$; CEA unknown for 66 patients. ${ }^{\circ}$ CYFRA $21-1$ : -, normal $(0.1-3.30 \mathrm{ng} / \mathrm{ml}) ;+$, elevated (>3.30 ng/ml); CYFRA 21-1 unknown for 110 patients. ${ }^{\mathrm{d}}$ Tumor staging was classified or reclassified according to the seventh edition of the International Union Against Cancer (UICC) staging system for Lung Cancer. ${ }^{\mathrm{e}}$ TACC 3 low expression, score $<5$; high expression, score $\geq 6$.

TACC3 expression is upregulated in primary NSCLC lesions compared with normal lung tissues. The present study indicated that high TACC3 expression in primary NSCLC lesions is correlated with poorer OS (25). We present that high TACC3 expression in primary NSCLC lesions is also associated with poorer RFS, which has not been reported. These data provide strong evidence for a relationship between high TACC3 expression levels and poor prognosis in NSCLC patients and indicate that the TACC 3 expression level is an independent prognostic factor for NSCLC patients. In addition, we compared the expression of TACC3 in metastatic lymph nodes tissues with normal lung tissues and demonstrated that expression of TACC 3 is also upregulated in metastatic lymph nodes tissues. These results indicate that alterations in TACC3 protein levels may be involved in both tumor development and progression.

We observed a strong correlation between more advanced clinical staging and higher T classification and higher TACC3 expression. These results may indicate a correlation between TACC3 overexpression and clinical progression in NSCLC. 


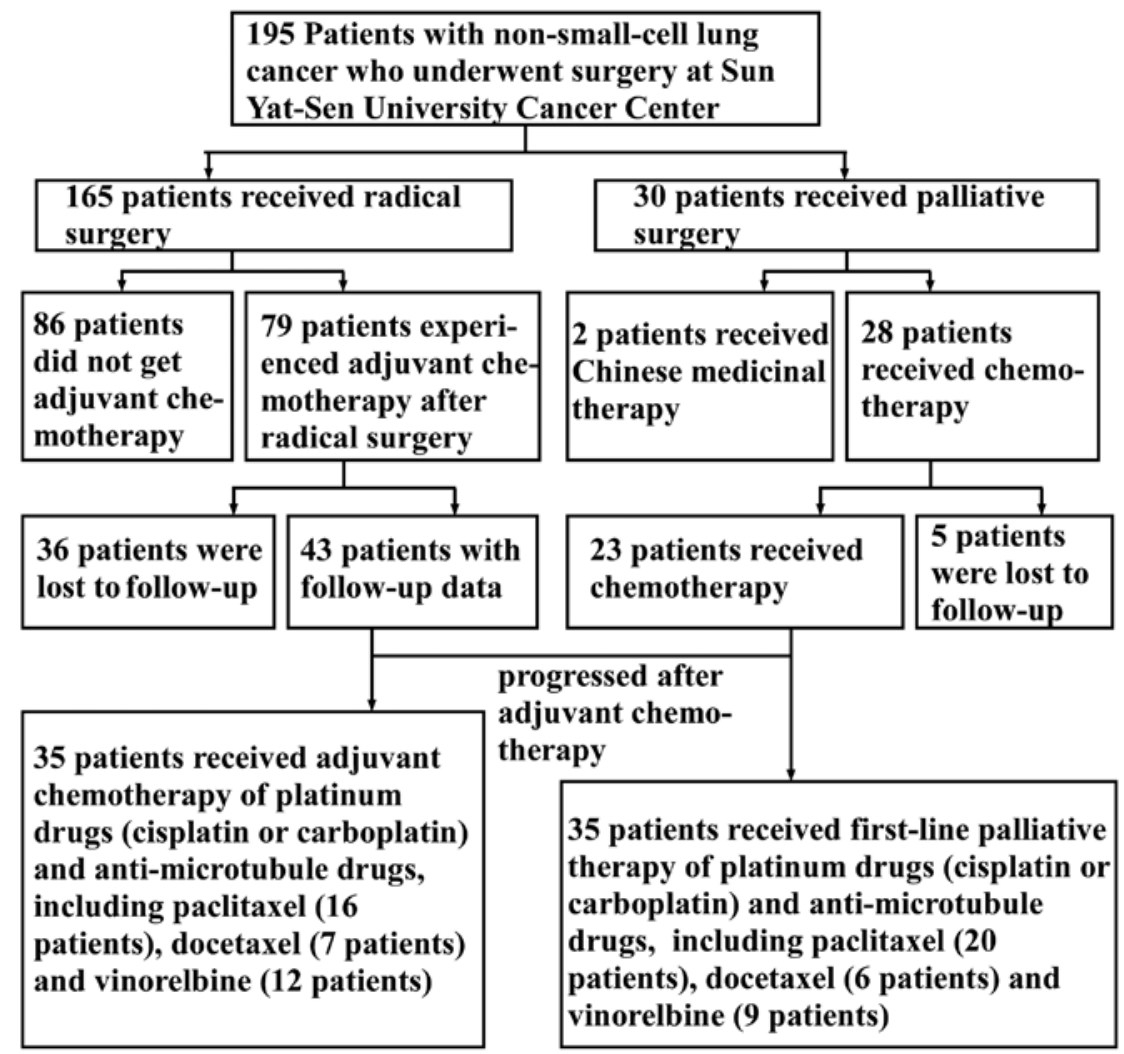

Figure 6 . Treatments received by patients enrolled in the present study.

Recent studies in cervical cancer have suggested that TACC3 overexpression promotes epithelial-mesenchymal transition (EMT) by activating the phosphatidylinositol 3-kinase (PI3K)/Akt and extracellular signal-regulated protein kinases (ERKs) signal transduction pathways $(32,33)$. As EMT plays a major role during cancer invasion and metastases, it is worthwhile to further investigate the role of TACC3 in the progression of NSCLC.

TACC 3 expression has been observed in various cell lines and tissues, particularly those undergoing rapid growth and differentiation. Sadek et al found that TACC3 is upregulated during the early differentiation of many varied cell types (17). Another study showed TACC3 plays an important role in murine embryonic development and may be involved in differentiation $(34,35)$. These observations showed the potential relationship between TACC 3 and differentiation stage in normal tissues. However, the correlation between TACC3 and the differentiation status in tumor tissues has not been studied to date. The present study demonstrated that high levels of TACC 3 expression were significantly correlated with poor differentiation in lung cancer, which had not been reported. Further experiments are needed to explore the molecular mechanisms of relationship between TACC3 expression and differentiation in tumor tissues.

Furthermore, the present study revealed that patients who smoked, squamous cell carcinoma patients and those with elevated CYFRA21-1 levels tend to have high expression levels of TACC3. More analysis is required to investigate the mechanisms underlying changes in TACC 3 expression in NSCLC patients.
The phosphorylation and localization of TACC 3 are novel pharmacodynamic indicators of Aurora A activity (36). Yim et al identified TACC 3 as a possible direct target of anti-microtubule drugs such as paclitaxel in cervical carcinoma cells (37). Subsequent studies have demonstrated that paclitaxel induces strong polyploidy and apoptosis in TACC3depleted murine fibroblast cells and that the downregulation of TACC3 enhances paclitaxel chemosensitivity in breast carcinoma cells $(38,39)$. However, if the expression of TACC3 in tumor tissue by immunohistochemical analysis correlates with the sensitivity to anti-microtubule drugs has not been reported yet. Our results indicate that low TACC3 expression may enhance chemosensitivity of anti-microtubule drugs in patients of lung cancer. However, larger samples ofpatients and further experiments in vitro are needed to verify the correlation between TACC 3 expression status and chemosensitivity to anti-microtubule drugs.

In conclusion, we demonstrated that TACC 3 expression is associated with the clinicopathological classification of NSCLC and that TACC3 is an independent prognostic factor for patient survival. In addition, TACC 3 overexpression may be associated with chemosensitivity to anti-microtubule drugs. Our findings suggest that the TACC 3 expression level may be a potential prognostic factor and a new therapeutic target for NSCLC.

\section{Acknowledgements}

The present study was supported by the National Scientific Foundation of China (no. 81372887), the National Basic 
Research Program of China (grant no. 2013CB910500), and the Guangdong Science and Technology Project (no. 2013B021800167).

\section{References}

1. Siegel R, Ma J, Zou Z and Jemal A: Cancer statistics, 2014. CA Cancer J Clin 64: 9-29, 2014

2. Gergely F: Centrosomal TACCtics. BioEssays 24: 915-925, 2002.

3. Peset I and Vernos I: The TACC proteins: TACC-ling microtubule dynamics and centrosome function. Trends Cell Biol 18: 379-388, 2008

4. Ha GH, Kim JL and Breuer EK: Transforming acidic coiled-coil proteins (TACCs) in human cancer. Cancer Lett 336: 24-33, 2013.

5. Albee AJ and Wiese C: Xenopus TACC3/maskin is not required for microtubule stability but is required for anchoring microtubules at the centrosome. Mol Biol Cell 19: 3347-3356, 2008.

6. van der Vaart B, Akhmanova A and Straube A: Regulation of microtubule dynamic instability. Biochem Soc Trans 37: 1007-1013, 2009.

7. Duensing S, Lee BH, Dal Cin P and Münger K: Excessive centrosome abnormalities without ongoing numerical chromosome instability in a Burkitt's lymphoma. Mol Cancer 2: 30-36, 2003.

8. Trachana V, van Wely KH, Guerrero AA, Fütterer A and Martínez-A C: Dido disruption leads to centrosome amplification and mitotic checkpoint defects compromising chromosome stability. Proc Natl Acad Sci USA 104: 2691-2696, 2007.

9. Gergely F, Karlsson C, Still I, Cowell J, Kilmartin J and Raff JW: The TACC domain identifies a family of centrosomal proteins that can interact with microtubules. Proc Natl Acad Sci USA 97: 14352-14357, 2000.

10. Raff JW: Centrosomes and cancer: Lessons from a TACC. Trends Cell Biol 12: 222-225, 2002.

11. Still IH, Vince P and Cowell JK: The third member of the transforming acidic coiled coil-containing gene family, TACC 3 , maps in 4p16, close to translocation breakpoints in multiple myeloma and is upregulated in various cancer cell lines. Genomics 58 $165-170,1999$.

12. Still IH, Hamilton M, Vince P, Wolfman A and Cowell JK: Cloning of TACC1, an embryonically expressed, potentially transforming coiled coil containing gene, from the $8 \mathrm{p} 11$ breast cancer amplicon. Oncogene 18: 4032-4038, 1999.

13. Cheng S, Douglas-Jones A, Yang X, Mansel RE and Jiang WG: Transforming acidic coiled-coil-containing protein 2 (TACC2) in human breast cancer, expression pattern and clinical/prognostic relevance. Cancer Genomics Proteomics 7: 67-73, 2010.

14. Conte N, Delaval B, Ginestier C, Ferrand A, Isnardon D, Larroque C, Prigent C, Séraphin B, Jacquemier J and Birnbaum D: TACC1-chTOG-Aurora A protein complex in breast cancer. Oncogene 22: 8102-8116, 2003.

15. Line A, Slucka Z, Stengrevics A, Li G and Rees RC: Altered splicing pattern of TACC1 mRNA in gastric cancer. Cancer Genet Cytogenet 139: 78-83, 2002.

16. Dhanasekaran SM, Barrette TR, Ghosh D, Shah R, Varambally S, Kurachi K, Pienta KJ, Rubin MA and Chinnaiyan AM: Delineation of prognostic biomarkers in prostate cancer. Nature 412: 822-826, 2001.

17. Sadek CM, Pelto-Huikko M, Tujague M, Steffensen KR, Wennerholm M and Gustafsson JA: TACC3 expression is tightly regulated during early differentiation. Gene Expr Patterns 3 : 203-211, 2003.

18. Conte N, Charafe-Jauffret E, Delaval B, Adélaïde J, Ginestier C, Geneix J, Isnardon D, Jacquemier $J$ and Birnbaum D Carcinogenesis and translational controls: TACC1 is down-regulated in human cancers and associates with mRNA regulators. Oncogene 21: 5619-5630, 2002.

19. Ma XJ, Salunga R, Tuggle JT, Gaudet J, Enright E, McQuary P, Payette T, Pistone M, Stecker K, Zhang BM, et al: Gene expression profiles of human breast cancer progression. Proc Natl Acad Sci USA 100: 5974-5979, 2003.

20. Lauffart B, Vaughan MM, Eddy R, Chervinsky D, DiCioccio RA, Black JD and Still IH: Aberrations of TACC1 and TACC 3 are associated with ovarian cancer. BMC Womens Health 5: 8-17, 2005 .
21. Peters DG, Kudla DM, Deloia JA, Chu TJ,Fairfull L, Edwards RP and Ferrell RE: Comparative gene expression analysis of ovarian carcinoma and normal ovarian epithelium by serial analysis of gene expression. Cancer Epidemiol Biomarkers Prev 14: $1717-1723,2005$.

22. Arlot-Bonnemains Y, Baldini E, Martin B, Delcros JG, Toller M, Curcio F, Ambesi-Impiombato FS, D'Armiento M and Ulisse S: Effects of the Aurora kinase inhibitor VX-680 on anaplastic thyroid cancer-derived cell lines. Endocr Relat Cancer 15: 559-568, 2008.

23. Duncan CG, Killela PJ, Payne CA, Lampson B, Chen WC, Liu J, Solomon D, Waldman T, Towers AJ, Gregory SG, et al: Integrated genomic analyses identify ERRFI1 and TACC 3 as glioblastoma-targeted genes. Oncotarget 1: 265-277, 2010.

24. Stewart JP, Thompson A, Santra M, Barlogie B, Lappin TR and Shaughnessy J Jr: Correlation of TACC3, FGFR3, MMSET and p21 expression with the $\mathrm{t}(4 ; 14)(\mathrm{p} 16.3 ; \mathrm{q} 32)$ in multiple myeloma. Br J Haematol 126: 72-76, 2004.

25. Jung CK, Jung JH, Park GS, Lee A, Kang CS and Lee KY: Expression of transforming acidic coiled-coil containing protein 3 is a novel independent prognostic marker in non-small cell lung cancer. Pathol Int 56: 503-509, 2006.

26. McShane LM, Altman DG, Sauerbrei W, Taube SE, Gion M and Clark GM; Statistics Subcommittee of the NCI-EORTC Working Group on Cancer Diagnostics: Reporting recommendations for tumor marker prognostic studies (REMARK). J Natl Cancer Inst 97: 1180-1184, 2005.

27. Kuang BH, Zhang MQ, Xu LH, Hu LJ, Wang HB, Zhao WF, $\mathrm{Du} \mathrm{Y}$ and Zhang $\mathrm{X}$ : Proline-rich tyrosine kinase 2 and its phosphorylated form pY881 are novel prognostic markers for non-small-cell lung cancer progression and patients' overall survival. Br J Cancer 109: 1252-1263, 2013.

28. Gangisetty O, Lauffart B, Sondarva GV, Chelsea DM and Still IH: The transforming acidic coiled coil proteins interact with nuclear histone acetyltransferases. Oncogene 23: 2559-2563, 2004.

29. Piekorz RP, Hoffmeyer A, Duntsch CD, McKay C, Nakajima H, Sexl V, Snyder L, Rehg J and Ihle JN: The centrosomal protein TACC3 is essential for hematopoietic stem cell function and genetically interfaces with p53-regulated apoptosis. EMBO J 21: 653-664, 2002

30. Richter JD and Theurkauf WE: Development. The message is in the translation. Science 293: 60-62, 2001.

31. Hood FE and Royle SJ: Pulling it together: The mitotic function of TACC3. BioArchitecture 1: 105-109, 2011

32. Ha GH, Park JS and Breuer EK: TACC3 promotes epithelialmesenchymal transition (EMT) through the activation of PI3K/ Akt and ERK signaling pathways. Cancer Lett 332: 63-73, 2013.

33. Ha GH, Kim JL and Breuer EK: TACC3 is essential for EGF-mediated EMT in cervical cancer. PLoS One 8: e70353, 2013.

34. Sadek CM,Jalaguier S,Feeney EP, Aitola M,Damdimopoulos AE, Pelto-Huikko M and Gustafsson JA: Isolation and characterization of AINT: A novel ARNT interacting protein expressed during murine embryonic development. Mech Dev 97: 13-26, 2000 .

35. McKeveney PJ, Hodges VM, Mullan RN, Maxwell P, Simpson D, Thompson A, Winter PC, Lappin TR and Maxwell AP: Characterization and localization of expression of an erythropoietin-induced gene, ERIC-1/TACC3, identified in erythroid precursor cells. Br J Haematol 112: 1016-1024, 2001.

36. LeRoy PJ, Hunter JJ, Hoar KM, Burke KE, Shinde V, Ruan J, Bowman D, Galvin K and Ecsedy JA: Localization of human TACC 3 to mitotic spindles is mediated by phosphorylation on $\mathrm{Ser}^{558}$ by Aurora A: A novel pharmacodynamic method for measuring Aurora A activity. Cancer Res 67: 5362-5370, 2007.

37. Yim EK, Tong SY, Ho EM, Bae JH, Um SJ and Park JS: Anticancer effects on TACC 3 by treatment of paclitaxel in HPV-18 positive cervical carcinoma cells. Oncol Rep 21: 549-557, 2009.

38. Schneider L, Essmann F, Kletke A, Rio P, Hanenberg H, SchulzeOsthoff K, Nürnberg B and Piekorz RP: TACC3 depletion sensitizes to paclitaxel-induced cell death and overrides p $21^{\mathrm{WAF}}$ mediated cell cycle arrest. Oncogene 27: 116-125, 2008.

39. Schmidt S, Schneider L, Essmann F, Cirstea IC, Kuck F, Kletke A, Jänicke RU, Wiek C, Hanenberg H, Ahmadian MR, et al: The centrosomal protein TACC 3 controls paclitaxel sensitivity by modulating a premature senescence program. Oncogene 29: 6184-6192, 2010. 